\title{
Instrumentos líticos del complejo pastoril temprano Chiu Chiu 200, norte de Chile
}

DONALD JACKSON $^{1}$ Y ANTONIA BENAVENTE $^{2}$

\begin{abstract}
RESUMEN
Este trabajo tiene por objetivo central caracterizar el instrumental lílico utilizado por una comunidad de pastores tempranos, localizada en el sitio de Chiu Chiu 200, Provincia de El Loa en el norte de Chile y datada hacia 900 AC. La caracterización del conjunto lítico intenta establecer singularidades técnico-subsistenciales de un incipiente modo de vida pastoril. Estas singularidades son establecidas en términos cualitativos y cuantitativos, a partir de una clasificación morfofuncional y del análisis de microhuellas de uso en los instrumentos líticos, considerando el conjunto artefactual asociado.
\end{abstract}

\begin{abstract}
The aim of this work is to characterize the lithic material used by the early shepherds community located on the Chiu Chiu 200 site, Loa Province, North of Chile dated by 900 BC. The description of the lithic set has as object to establish the technical-subsistence characteristics of an incipient way of shepherd life. These characteristic are stablished by qualitative and quantitative terms, from a morphofunctional clasification and from the wear-use analysis of the lithic material considering the associated material set.
\end{abstract}

\section{Introducción}

El presente estudio tiene por propósito caracterizar el componente lítico de un campamento de pastores tempranos denominado Chiu Chiu 200, situado en la Provincia de El Loa, en el norte de Chile. Se intenta establecer en términos cualitativos y cuantitativos el equipo de instrumental lítico propio de una comunidad pastoril.

Para el propósito anterior se ha utilizado la totalidad de una muestra lítica recuperada en una excavación de $60 \mathrm{~m}^{2}$ que proporcionaron 1503 instrumentos. El análisis de este conjunto se realizó a base de una clasificación morfofuncional y al examen microscópico de las microhuellas de uso, lo que permitió caracterizar el conjunto estudiado, definiendo ca-

1 Departamento de Antropología, Universidad de Chile, Santiago, CHILE. tegorías y sus frecuencias relacionándolas con la economía del grupo.

Se expone en primer lugar el marco teórico-metodológico a base del cual se estudió el material, luego se describen las categorías de instrumentos identificados, para terminar discutiendo las implicancias tecnoeconómicas y funcionales de los instrumentos líticos de la comunidad pastoril del sitio de donde proceden.

\section{Marco teórico-metodológico}

El origen y desarrollo de la vida pastoril en el norte de Chile, aunque ha sido preocupación de los arqueólogos, sólo recientemente se han conducido trabajos sistemáticos encauzados a definir el problema (Benavente 1981; Núñez 1983). Excavaciones en sitios claves como el estudio de los materiales recuperados podrán ir vislumbrando las características y singularidades del proceso.

Respecto a lo anterior, uno de los problemas es que las diferencias en el registro arqueológico entre sitios cazadores y pastoriles no son evidentes, sin embargo, establecer estas diferencias parece ser una tarea básica y previa para conocer la naturaleza del proceso. En este sentido el estudio del instrumental lítico debiera reflejar diferencias respecto a la base económica, distinguiendo el equipo instrumental propio de grupos pastoriles. En esta perspectiva, el presente estudio tiene por objetivo, por una parte, caracterizar el equipo de instrumentos líticos de la comunidad pastoril estudiada y, por otra, la inferencia de algunos aspectos del modo de vida que implica esta forma de subsistencia.

Considerando lo anterior, se ha seleccionado una muestra lítica del sitio arqueológico de Chiu Chiu 200, el cual se encuentra situado próximo a la localidad del mismo nombre, en la Provincia de El Loa (II Región). Inicialmente este sitio fue definido como un poblado agroalfarero temprano (Benavente 1978), sin embargo, posteriores investigaciones han permitido ampliar y precisar la naturaleza cultural del 
sitio definiéndolo como un campamento de pastores tempranos (Benavente 1981).

Las excavaciones de $60 \mathrm{~m}^{2}$ en el sitio han permitido observar que se trata de un campamento habitacional con estructuras semicirculares datado en 910 \pm 290 años AC, a la cual se asocian "muros basureros" en donde se ha registrado un amplio componente artefactual que incluye textiles, cestería, cerámica, artefactos varios además de la lítica, y restos ecofactuales que incluyen huesos de animales, cuero, moluscos, restos de madera, vegetales y plumas. Las características del sitio y sus materiales hablan en favor de un campamento de pastores tempranos para el norte de Chile (Benavente 1982).

Las muestras líticas recuperadas en las excavaciones fueron estudiadas en su totalidad (1503 piezas), las cuales fueron parcialmente limpiadas, luego de lo cual fueron rotuladas. Durante estos procesos y otros, se trató en lo posible de evitar el deterioro del material de tal forma de no destruir los microindicios del uso o confundirlos con alteraciones provocadas durante la manipulación de los instrumentos. A este respecto se consideraron los procesos que pudieron actuar durante la recuperación y análisis de la muestra, así como aquellos efectos tecnológicos y depositacionales (Jackson 1989).

Como el propósito del estudio fue determinar el equipo instrumental que caracteriza un modo de vida pastoril, se enfatizó en un análisis funcional de los instrumentos. Para esto se procedió primero, a una clasificación morfofuncional (Bate 1971) determinando categorías genéricas funcionales, las que fueron subdivididas según variedades morfológicas; así, por ejemplo, se distinguió raspadores y enseguida su variedad morfológica; convexos, terminales, etc. Esta misma clasificación fue ampliada y precisada a base del examen microscópico de las huellas de uso.

Para el examen microscópico se utilizó una Lupa Stereoscópica NIKON (SM2-10/Tipo 102) con un ocular de $15 \mathrm{x}$ y objetivo de 0.66 a $4 \mathrm{x}$, lo que permitió un examen de bajo aumento orientado a determinar presencia de microhuellas, tipos y en algunos casos observar características diferenciales que pudieran hablarnos sobre la naturaleza de los materiales trabajados. Se utilizó en el examen luz indirecta por medio de una lámpara NIKON con un voltaje de 3 a 8 v que permitía orientar y focalizar la luz. Los instrumentos fueron lavados con alcohol previo a un ligero examen para evitar la destrucción de residuos orgánicos (Briuer 1976), luego se examinaban los bordes más detenidamente con luz en ángulo rasante y/o variando el ángulo del instrumento examinado.

Del total de la categoría identificada como derivados de núcleo sin modificaciones intencionales se seleccionó una muestra en forma aleatoria y estratificada para someterla al examen de microhuellas de uso para determinar si se trataba de instrumentos de filos vivos. El examen microscópico mostró que un porcentaje importante de la muestra seleccionada efectivamente correspondía a instrumentos de filos vivos.

Las piezas con microhuellas de uso fueron clasificadas según la forma y ángulo del borde activo, el ángulo se midió con un goneómetro de contacto (13 W0561), la forma del borde se definió en términos geométricos: convexo, cóncavo, recto e irregular. Luego se aplicó la variable extensión de las microhuellas; marginal, bimarginal, etc., observando el tipo de microhuellas y en caso de existir sus características diferenciales. En este proceso de clasificación se tomaron como variable rectora las características únicamente del borde activo identificado por las huellas de uso y en lo particular se dio más importancia al ángulo del borde como variable más relevante para definir categorías genéricas de función (Wilmsen 1968).

Para definir, caracterizar e interpretar las microhuellas se han utilizado las sugerencias y proposiciones de Gould y colaboradores (1971), Tringham y colaboradores (1974), Keeley (1980), Odell y Odell (1980), Odell (1980) y Semenov (1982).

Los resultados del análisis se han expuesto en términos de descripciones verbales, histogramas de frecuencia y gráficas que resumen la caracterización de la muestra estudiada. Por otra parte, se ha dibujado la mayoría de las categorías líticas identificadas y se tomaron microfotografías de algunas piezas que mostraban microhuellas de uso que atestiguaban su utilización.

\section{Resultados: Categorías líticas identificadas:}

Las categorías identificadas morfofuncionalmente se han subdividido de acuerdo al criterio tecnológico 


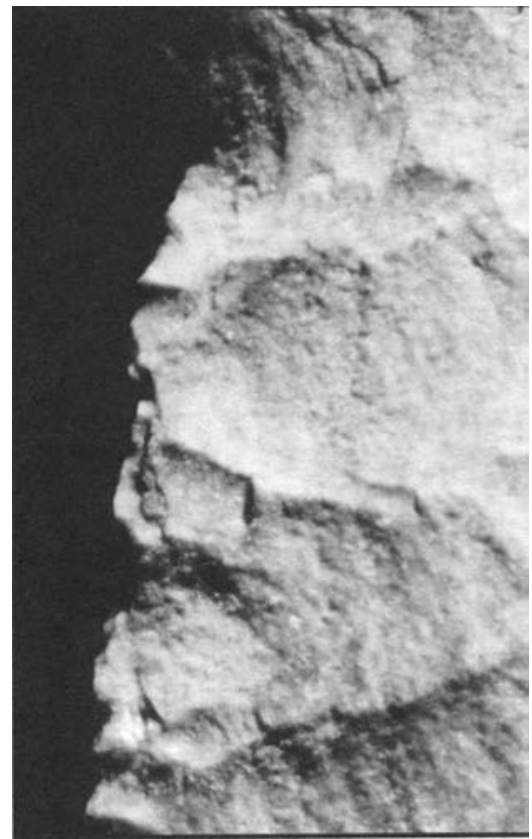

Figura 1. Borde retocado de cuchillo bifacial con microastillamiento por uso $(15 \times 0.66 \mathrm{x})$.

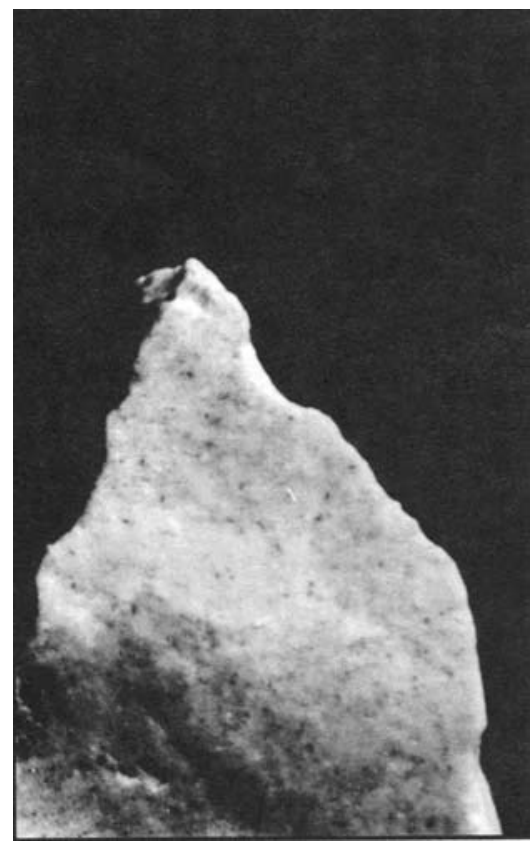

Figura 3. Extremo de lasca con golpe de buril y microastillamiento producto de la acción de burilado $(15 \mathrm{x} 0.66 \mathrm{x})$.

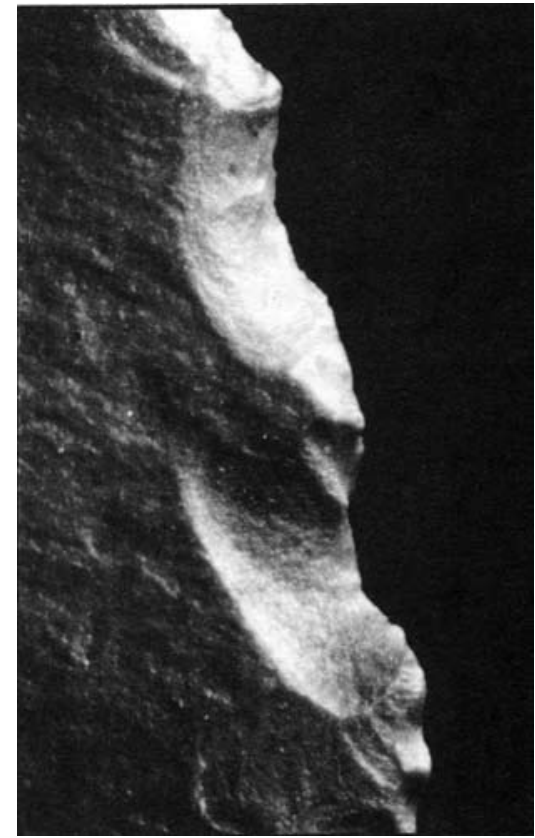

Figura 2. Borde de lasca de filo vivo con microastillamiento y desgaste indicativa de un cuchillo $(15 \times 0.66 \mathrm{x})$.

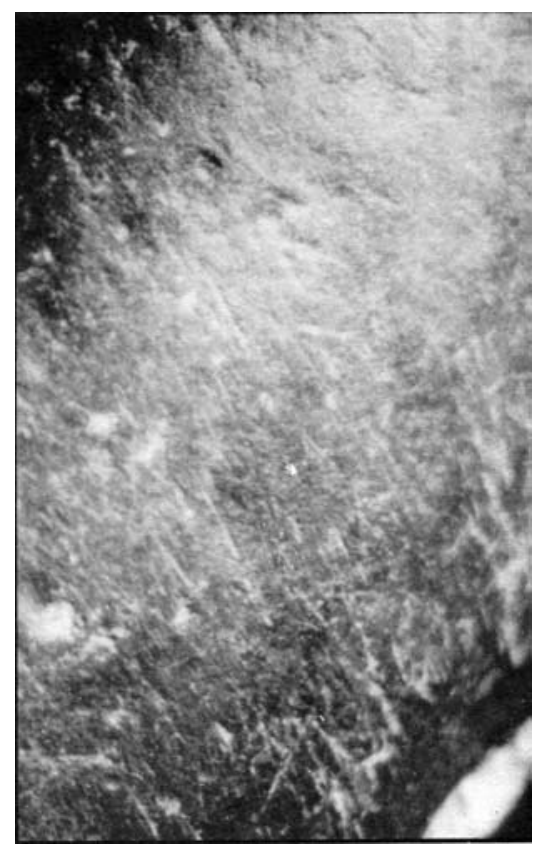

Figura 4. Superficie de guijarro con claras estrías y desgaste por uso producto de la utilización como pulidor de cerámica $(15 \mathrm{x} 0.66 \mathrm{x})$. 
en dos grupos, por una parte la lítica tallada y por otra la lítica pulida-piqueteada. Un grupo distinto se encuentra constituido por instrumentos de filos vivos identificados por la presencia de microhuellas de uso observadas microscópicamente.

Las categorías de la lítica tallada incluye: 1) Puntas de proyectiles triangulares apedunculadas $(0.21 \%)$; 2) Raederas laterales convexas (0.77\%); 3) Cuchillos discoidales, semidiscoidales, bifaciales lanceolados y en pieza retomada $(0.98 \%)$; 4) Cuchillo-raspador $(0.28 \%)$; 5) Raspadores irregulares, cóncavos, laterales convexos y cóncavo de dorso alto, sobre trozos irregulares abultados, cóncavos sobre matriz ancha, laterales, convexos terminales, de borde activo convexo y de perfil cóncavo-convexo, de borde activo escotado y atípicos $(3.94 \%)$; 6) Cepillos $(0.70 \%)$; 7) Macroperforadores, microperforadores y perforadores en piezas retomadas (1.12\%); 8) Grabadores $(0.07 \%)$; 9) Buriles $(0.21 \%)$; 10$)$ Cinceles $(0.07 \%)$; 11) Cuñas $(2.11 \%)$; 12) Percutor-cincel $(0.07 \%)$; 13) Cinceles y/o cuñas bipolares $(0.14 \%)$; 14$)$ Lascas modificadas intencionalmente de función no definible; marginal simple, marginal doble, marginal simple-opuesto, bimarginal simple, bimarginal doble-simple, bimarginal doble, facial marginal doble y con astillamiento atípico $(5.06 \%) ; 15)$ Tajadores bilaterales y perimetrales $(1.47 \%)$; 16) Cantos astillados $(2.39 \%)$; 17) Materia prima o trozos astillados (0.35\%); 18) Preformas (1.97\%); 19) Núcleos $(2.74 \%)$; 20) Trozos nucleiformes utilizados como instrumentos $(9.00)$; 21) Derivados de núcleo sin modificaciones intencionadas (63.23\%) y Percutores (1.26) (Figura 5).

Las categorías identificadas en la lítica pulida-piqueteada incluye: 1) Manos de moler cuadrangulares, rectangulares y ovoidales (32.92\%); 2) Piedras de moler y morteros $(8.53 \%)$; 3) Fragmentos de palas (21.95\%); 4) Desgastadores abrasivos (3.65\%); 5) Pulidores para cerámica (2.21\%); 6) Instrumentos sobre piedra pómez; seudotorteras, "tapones", preformas y otras piezas de función no definida (24.39\%), y 7) Instrumentos abrasivos sobre arenisca (7.31\%) (Figura 6).

Un gran número de estas categorías de instrumentos morfofuncionalmente definidos mostraron claros indicios de microhuellas de utilización, presentándose en orden de su frecuencia microastillamiento, desgaste, pulido, estriamiento y en algunos casos presuntos residuos orgánicos productos del uso. Las características de las microhuellas sugieren un uso intenso de tales instrumentos, en su mayoría utilizados sobre materiales blandos (cuero, carne y vegetales) y en menor proporción sobre materiales duros (hueso, concha y otros).

El tercer grupo se encuentra constituido por aquellos instrumentos de filos vivos. Del total de 906 derivados de núcleo sin modificaciones intencionales, se seleccionó una muestra en forma aleatoria estratificada para análisis microscópico para detectar microhuellas de uso. La muestra seleccionada correspondió a un total de 107 piezas, es decir, $11.81 \%$ del total.

Los resultados del análisis muestran que 52.20\% (58 piezas) de la muestra examinada microscópicamente presentaba microhuellas de uso, las restantes, 45.79\% no presentaban indicios de utilización. Los tipos de microhuellas detectadas fueron los siguientes: microastillamiento (67.24\%), microastillamiento y desgaste $(27.58 \%)$, desgaste (1.72\%), microastillamiento y residuos (1.72\%), y microastillamiento, desgaste y pulido (1.72\%). No se distinguieron características diferenciales del astillamiento por uso, aunque se observaron diferencias significativas que reflejan tanto distintas funciones y usos específicos.

El 77.58\% de las lascas con huellas de uso presentaba sólo un borde utilizado, lo que de acuerdo al ángulo, forma del borde y extensión de las microhuellas fueron clasificadas en instrumentos de corte; cuchillos $(11.11 \%)$, instrumentos de corte-desgaste; raederas y/o cuchillos (51.11\%) e instrumentos de desgaste; raspadores, cepillos y otros $(40.00 \%)$.

Las lascas con dos bordes utilizados incluyen $22.41 \%$, fueron clasificadas de la misma forma que el grupo anterior y es posible observar que en su mayoría corresponden a instrumentos multifuncionales o polivalentes.

En términos de categorías funcionales es posible asegurar la existencia de cuchillos, raederas-cuchillos, raederas y raspadores, además de instrumentos multifuncionales. Respecto a los usos específicos, las huellas sugieren un alto porcentaje de lascas utilizadas sobre materiales blandos desde madera a carne y una baja frecuencia para el trabajo de materiales duros como el hueso.

Las características y frecuencia de este tipo de instrumentos sugieren un destino no especializado, donde los instrumentos luego de ser usados fueron desechados, reemplazados por otros similares, 


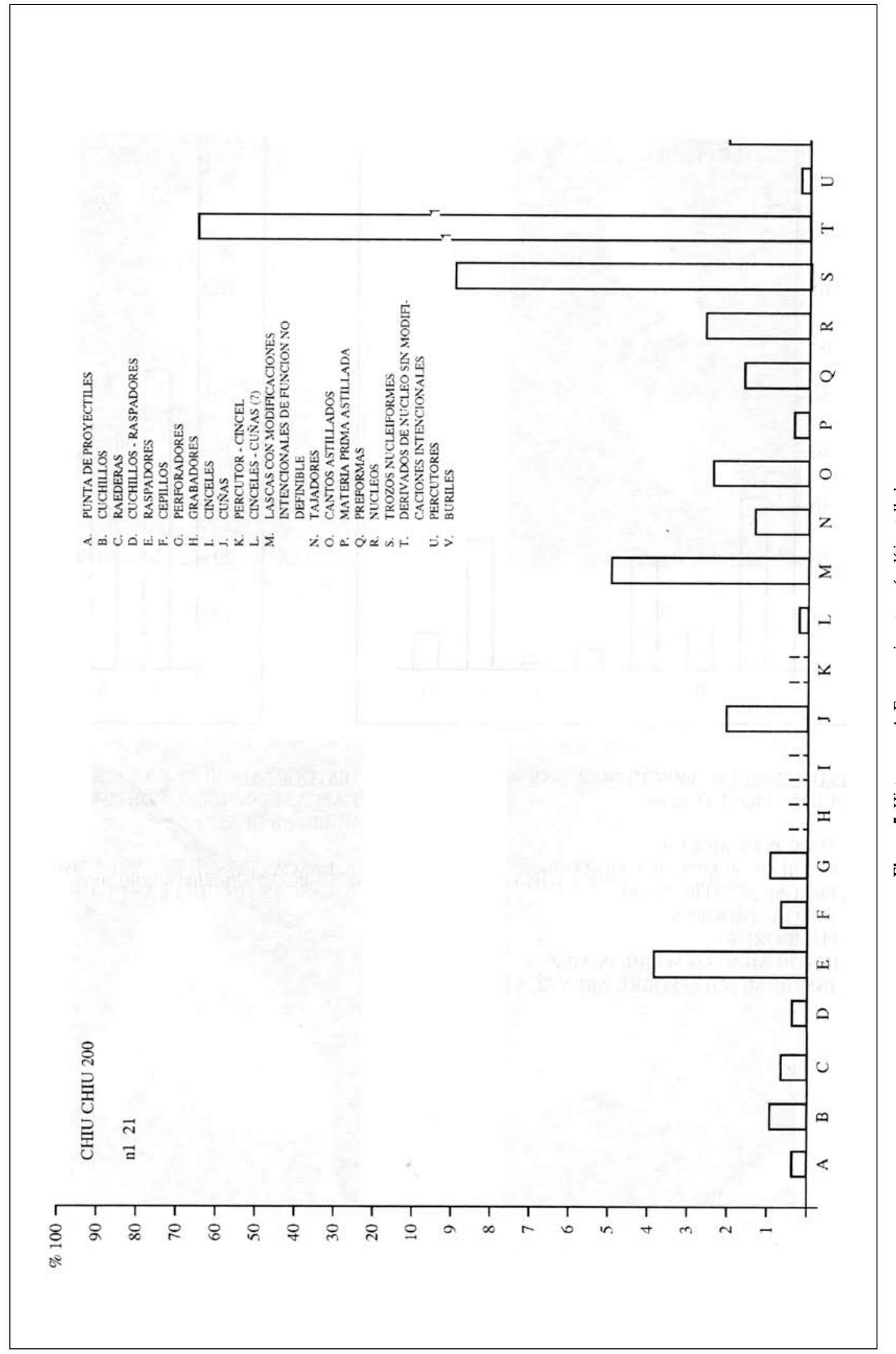




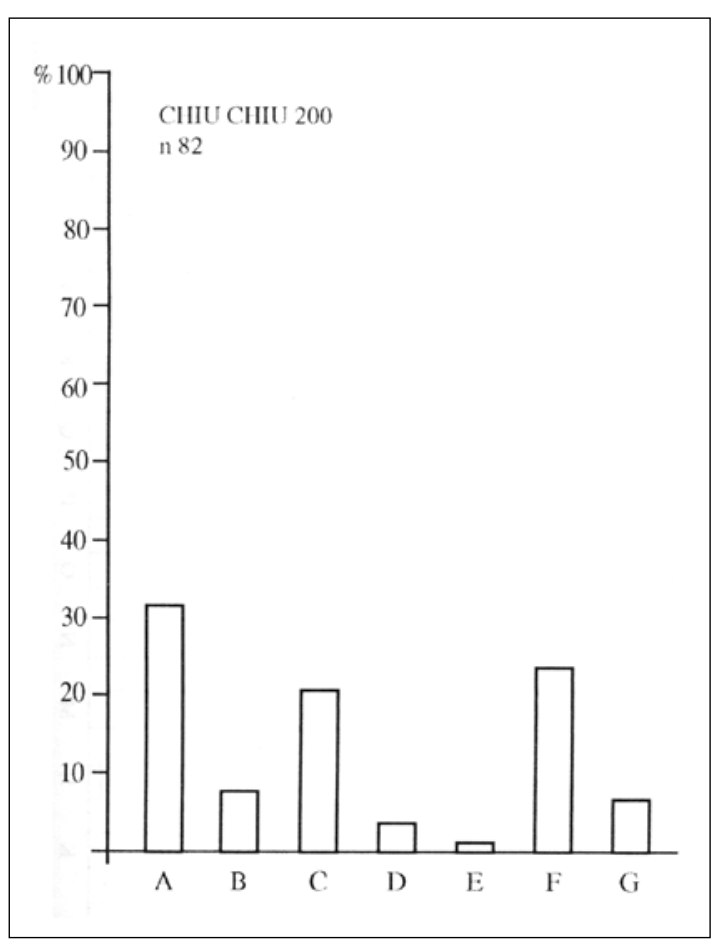

Figura 6. Histograma 2: Frecuencia lítica pulida - piqueteada a. Manos de Moler

b. Piedras de Moler y Morteros

c. Fragmentos de Palas

d. Desgastadores

e. Pulidores

f. Instrumentos sobre Pómez

g. Instrumentos sobre Arenisca

aprovechándose la eficiencia de los filos vivos. Algún porcentaje de lascas donde no se detectaron huellas de uso es posible que fueran utilizadas muy ligeramente sobre materiales muy blandos, el resto, sin embargo, debe ser considerado desechos o bien instrumentos potenciales incluso no tallados localmente, sino trasladados al sitio para eventualmente ser utilizados.

\section{Discusión y conclusiones}

En primer lugar, debemos señalar que el procedimiento empleado en el análisis nos ha permitido en términos morfofuncionales definir un gran número de categorías funcionales, las que han podido ser precisadas a base del estudio de las microhuellas de uso que estos instrumentos formalizados presentaban. Sin embargo, lo importante de recalcar es el análisis microscópico para la detección de huellas de uso en aquellos derivados de núcleo, lascas y láminas, sin modificaciones intencionales,

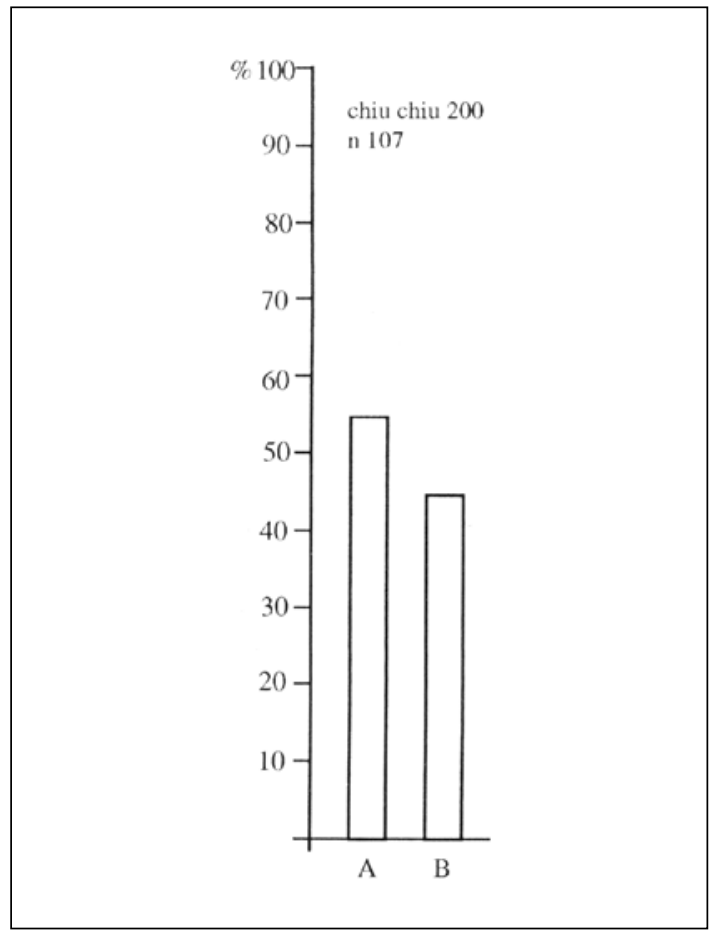

Figura 7. Histograma 3: Frecuencia de lascas con huellas de uso y sin huellas de uso

a. Lascas con huellas de uso

b. Lascas sin huellas de uso

mostrar que un gran número de ellas no sólo deben ser clasificadas como subproductos del proceso de talla o desechos, sino que se trata de instrumentos de filos vivos que no son posibles de definir sólo a partir de un análisis morfológico. Esto tiene dos implicancias metodológicas e interpretativas de relevancia, por una parte el análisis de microhuellas de uso permite definir instrumentos no formalizados que morfológicamente no es posible distinguir $\mathrm{y}$, por otra, esto implica, en consecuencia, que el análisis microscópico en lascas de filos vivos permite ampliar y precisar en términos de las categorías funcionales identificadas y sus respectivas frecuencias el conjunto de instrumentos utilizados por un grupo o identificado en un sitio. En caso contrario, la identificación de instrumentos sólo a base morfofuncionales implicaría un sesgo cualitativo y principalmente cuantitativo que en algunos casos pueden ser definitorios para caracterizar y distinguir un conjunto lítico de otro. Las implicancias interpretativas de ello son evidentes.

El porcentaje de lítica tallada corresponde a $94.54 \%$ y el de la lílica pulida-piqueteada a $5.45 \%$, 
diferencia que normalmente se da en la mayoría de los sitios; sin embargo hay que destacar que en la lítica pulida hay un alto porcentaje de implementos de molienda no comparable a la frecuencia que se da en sitios netamente de cazadores recolectores, lo que sugiere una mayor dependencia de vegetales. También en la lítica pulida se da una serie de implementos diversos principalmente instrumentos abrasivos que debieron ser utilizados para el trabajo de materiales blandos, a este respecto trozos de pómez podrían estar vinculados para el tratamiento de cuero, lo mismo tal vez algunas manos de moler como se ha sabido de su utilización para este fin a partir de referentes etnográficos (Adams 1988). En lo general las categorías de la lítica pulida-piqueteada están mostrando un conjunto instrumental distinto al típicamente de cazadores recolectores y al mismo tiempo categorías que podrían ser vinculadas con una dependencia más que nada agroganadera.

Respecto a la frecuencia de las categorías de instrumentos retocados, llama inmediatamente la atención la baja frecuencia de puntas de proyectiles respecto a cuchillos y raederas utilizados para el destazamiento de animales. La frecuencia de instrumentos de corte destinados para este tipo de uso se incrementa aún más si se considera la frecuencia de lascas utilizadas como instrumentos de corte con filos vivos, todo lo cual está mostrando que estamos frente a una comunidad no cazadora, pero sí dependiendo de productos cárneos de camélidos los que fueron procesados con los instrumentos de corte. Esto además ha quedado atestiguado directamente en las marcas de cuchillo evidenciadas en algunas osamentas de camélidos.

Los raspadores se presentan con una alta frecuencia y variabilidad morfológica, además de lascas de filos vivos utilizadas para esta función. La amplia variedad morfológica sugiere destinos diversos entre los cuales debe considerarse el trabajo de la preparación de cuero.

Otra categoría con una alta frecuencia en el sitio corresponde a las cuñas y cinceles, pocas veces identificados en los reportes arqueológicos, tal vez por una falta de detección de sus rasgos característicos que lo definen; en cualquier caso parece ser una categoría nueva y frecuente relacionada funcionalmente con el trabajo de la madera.

Los tajadores y cantos astillados representan también una relativa alta frecuencia vinculable funcionalmente con el procesamiento de madera. Por otra parte, núcleos y trozos nucleiformes fueron reutilizados en gran número en tareas de corte por percusión y machacamiento con indicios claros de percusión que es tentativamente relacionable con la fractura de huesos largos para extraer la médula y astillas de hueso presuntamente utilizables.

La alta frecuencia de instrumentos de filos vivos destinados a distintas funciones sugieren tareas diversificadas en donde no se requirió adecuar la forma y ángulo de los bordes por medio del retoque. A este respecto la alta frecuencia de núcleos y derivados de núcleo sin modificaciones intencionales sugieren un procesamiento local de un gran número de materias primas, otras, en cambio, en menor proporción, fueron llevadas al sitio en forma semiprocesada o bien ya como instrumentos finiquitados. Sin hacer una comparación particular respecto a algún sitio de cazadores, hay evidencias claras tanto cualitativas como frecuenciales que muestran que el instrumental lítico no responde a una economía cazadora, sino claramente agroganadera, vinculándose algunas categorías funcionales y frecuencias significativas con tareas de un grupo pastoril. Una futura comparación con muestras estudiadas bajo la misma metodología procedentes de sitios de una base económica distinta nos permitirá con precisión establecer diferencias categoriales y frecuenciales en el instrumental lítico utilizado por grupos de base subsistenciales distintas.

\section{Agradecimientos}

Este trabajo fue financiado por FONDECYT (Proyecto 792-89). Comprometen nuestra gratitud Claudio Massone, quien gentilmente colaboró en el procedimiento de muestreo para seleccionar el material que fue estudiado microscópicamente, y Rodrigo Sánchez, quien colaboró en el rotulado y procesamiento inicial de los materiales estudiados. 


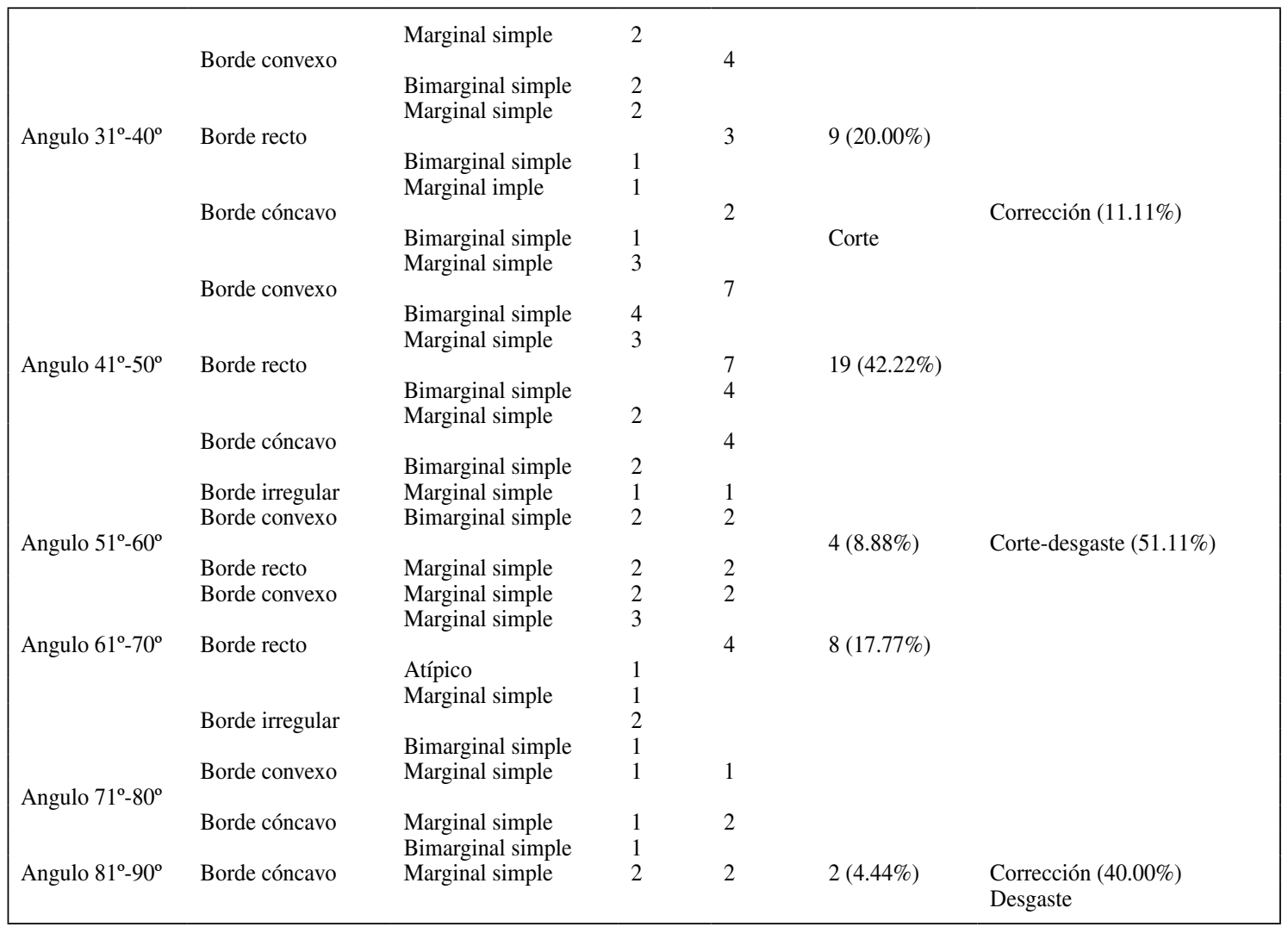

Tabla 1. Lascas con un borde utilizado; relación ángulo, forma y extensión microhuellas (45/77.58\%)

\begin{tabular}{|c|c|c|c|c|c|}
\hline Angulo & $\begin{array}{l}\text { Izquierdo } 35^{\circ} \\
\text { Derecho } 36^{\circ}\end{array}$ & $\begin{array}{l}\text { convexo } \\
\text { irregular }\end{array}$ & $\begin{array}{l}\text { Marginal simple } \\
\text { Marginal simple }\end{array}$ & $\begin{array}{l}1 \\
1\end{array}$ & Unifuncional \\
\hline Angulo & $\begin{array}{l}\text { Izquierdo } 60^{\circ} \\
\text { Derecho }\end{array}$ & $\begin{array}{l}\text { convexo } \\
\text { cóncavo }\end{array}$ & $\begin{array}{l}\text { Marginal simple } \\
\text { Marginal simple }\end{array}$ & $\begin{array}{l}1 \\
1\end{array}$ & \\
\hline Angulo & $\begin{array}{l}\text { Izquierdo } 46^{\circ} \\
\text { Derecho } 36^{\circ}\end{array}$ & $\begin{array}{l}\text { convexo } \\
\text { convexo }\end{array}$ & $\begin{array}{l}\text { Marginal simple } \\
\text { Marginal simple }\end{array}$ & $\begin{array}{l}1 \\
1\end{array}$ & Unifuncional \\
\hline Angulo & $\begin{array}{l}\text { Izquierdo } 44^{\circ} \\
\text { Derecho } 60^{\circ}\end{array}$ & $\begin{array}{l}\text { convexo } \\
\text { cóncavo }\end{array}$ & $\begin{array}{l}\text { Marginal simple } \\
\text { Marginal simple }\end{array}$ & $\begin{array}{l}1 \\
1\end{array}$ & \\
\hline Angulo & $\begin{array}{l}\text { Izquierdo } 57^{\circ} \\
\text { Derecho } 53^{\circ}\end{array}$ & $\begin{array}{l}\text { recto } \\
\text { irregular }\end{array}$ & $\begin{array}{l}\text { Marginal simple } \\
\text { Bimarginal simple }\end{array}$ & $\begin{array}{l}1 \\
1\end{array}$ & \\
\hline Angulo & $\begin{array}{l}\text { Izquierdo } 55^{\circ} \\
\text { Derecho } 52^{\circ}\end{array}$ & $\begin{array}{l}\text { recto } \\
\text { recto }\end{array}$ & $\begin{array}{l}\text { Marginal simple } \\
\text { Marginal simple }\end{array}$ & $\begin{array}{l}1 \\
1\end{array}$ & Unifuncional \\
\hline Angulo & $\begin{array}{l}\text { Izquierdo } 47^{\circ} \\
\text { Derecho } 64^{\circ}\end{array}$ & $\begin{array}{l}\text { cóncavo } \\
\text { convexo }\end{array}$ & $\begin{array}{l}\text { Marginal simple } \\
\text { Marginal simple }\end{array}$ & $\begin{array}{l}1 \\
1\end{array}$ & \\
\hline Angulo & $\begin{array}{l}\text { Izquierdo } 38^{\circ} \\
\text { Derecho } 27^{\circ}\end{array}$ & $\begin{array}{l}\text { recto } \\
\text { irregular }\end{array}$ & $\begin{array}{l}\text { Bimarginal simple } \\
\text { Bimarginal simple }\end{array}$ & $\begin{array}{l}1 \\
1\end{array}$ & \\
\hline Angulo & $\begin{array}{l}\text { Izquierdo } 41^{\circ} \\
\text { Derecho } 60^{\circ}\end{array}$ & $\begin{array}{l}\text { convexo } \\
\text { cóncavo }\end{array}$ & $\begin{array}{l}\text { Bimarginal simple } \\
\text { Bimarginal simple }\end{array}$ & $\begin{array}{l}1 \\
1\end{array}$ & \\
\hline Angulo & $\begin{array}{l}\text { Izquierdo } 58^{\circ} \\
\text { Derecho } 65^{\circ}\end{array}$ & $\begin{array}{l}\text { convexo } \\
\text { recto }\end{array}$ & $\begin{array}{l}\text { Bimarginal simple } \\
\text { Bimarginal simple }\end{array}$ & $\begin{array}{l}1 \\
1\end{array}$ & \\
\hline Angulo & $\begin{array}{l}\text { Izquierdo } 42^{\circ} \\
\text { Derecho } 50^{\circ}\end{array}$ & $\begin{array}{l}\text { convexo } \\
\text { recto }\end{array}$ & $\begin{array}{l}\text { Bimarginal simple } \\
\text { Marginal simple }\end{array}$ & $\begin{array}{l}1 \\
1\end{array}$ & \\
\hline Angulo & $\begin{array}{l}\text { Izquierdo } 40^{\circ} \\
\text { Derecho } 54^{\circ}\end{array}$ & $\begin{array}{l}\text { irregular } \\
\text { recto }\end{array}$ & $\begin{array}{l}\text { Marginal simple } \\
\text { Marginal simple }\end{array}$ & $\begin{array}{l}1 \\
1\end{array}$ & \\
\hline Angulo & $\begin{array}{l}\text { Izquierdo } 50^{\circ} \\
\text { Derecho } 40^{\circ}\end{array}$ & $\begin{array}{l}\text { convexo } \\
\text { recto }\end{array}$ & $\begin{array}{l}\text { Bimarginal simple } \\
\text { Marginal simple }\end{array}$ & $\begin{array}{l}1 \\
1\end{array}$ & \\
\hline
\end{tabular}

Tabla 2. Lascas con dos bordes utilizados; relación ángulo, forma y extensión microhuellas (13/22.41\%) 

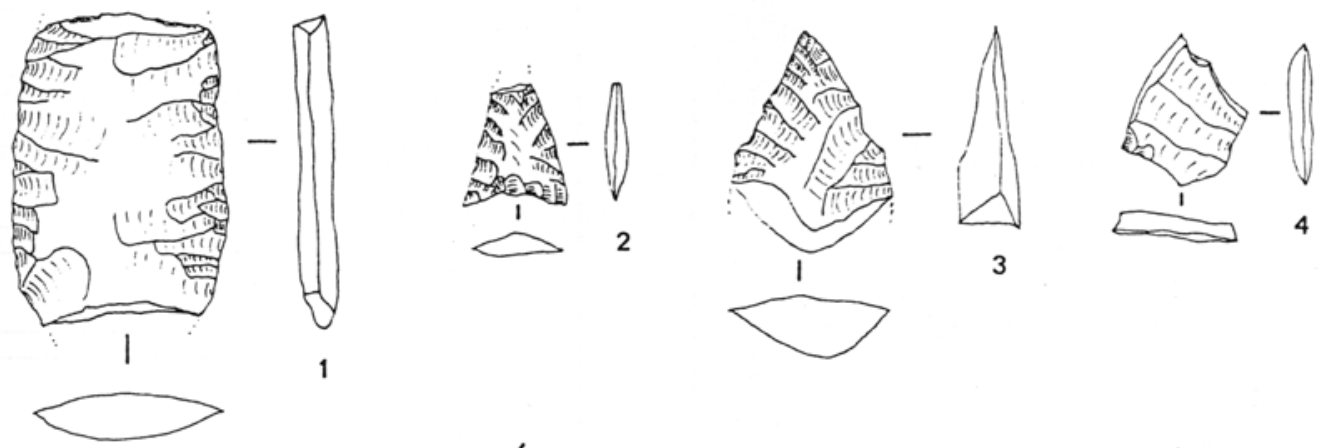

3
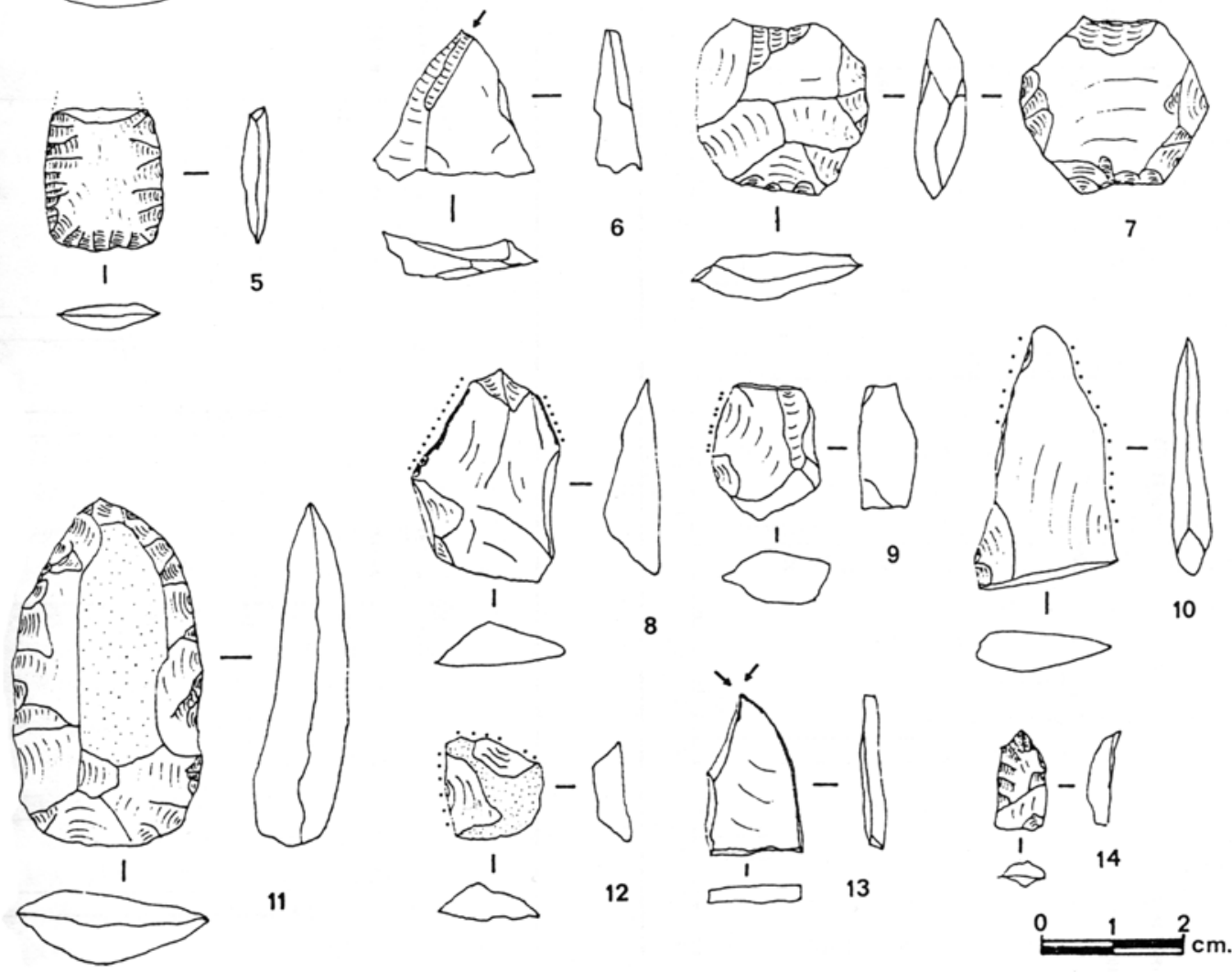

Figura 8. Artefactos líticos.

1. Cuchillo bifacial con extremo de fractura reutilizada

2. Punta de proyectil triangular apedunculada

3. Extremo distal de punta de proyectil

4. Perforador de cuerpo diferenciado

5. Extremo proximal - medial de punta de proyectil

6. Buril

7. Cuña $y / o$ cincel

8. Lasca de filo vivo utilizada para raspar

9. Lasca de filo vivo utilizada para raspar

10. Lasca de filo vivo utilizada para cortar

11. Preforma posible de cuchilo

12. Lasca de filo vivo utilizada para raspar

13. Buril de dos extracciones

14. Grabador 
DONALD JACKSON, ANTONIA BENAVENTE

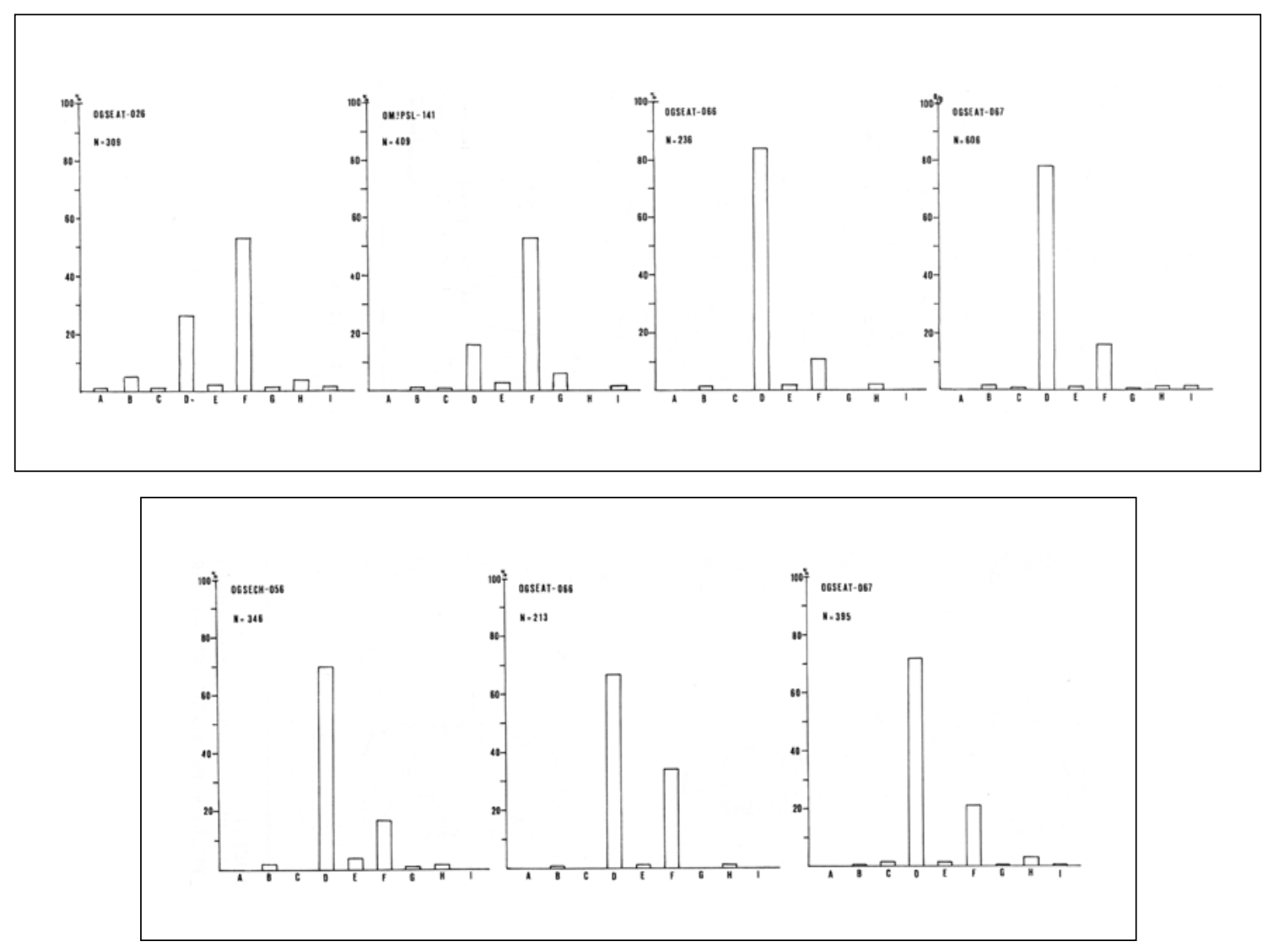

Figura 9. Gráficos.

44 


\section{REFERENCIAS CITADAS}

ADAMS, J., 1988. Use-wear anallysis on manos and hide-processing stone. Journal of Field Arqueology 15 (3).

BATE, L. F., 1971. Material lítico: Metodología de clasificación. Noticiario Mensual del Museo Nacional de Historia Natural XVI 181-182.

BENAVENTE, A., 1978. Chiu Chiu 200: Poblado agroalfarero temprano. Revista Chilena de Antropología 1.

_ 1981. Chiu Chiu 200: Un campamento de pastores. Tesis para optar al Grado de Licenciado en Arqueología y Prehistoria. Departamento de Ciencias Sociológicas y Antropológicas, Universidad de Chile, Santiago.

_ 1982. Chiu Chiu 200: Una comunidad pastora temprana en la Provincia de El Loa (II Región). Actas del IX Congreso Nacional de Arqueología. La Serena.

- 1988Ms. Nuevas evidencias arqueológicas acerca de los asentamientos tempranos en el Loa Medio.

BRIUER, F., 1976. New clue to stone tool function; plant animal residues. American Antiquity 41 (4).

GOULD, R., D. KOSTER y A. SONTZ, 1971. The lithic asemblage of the western desert aborigines of Australia. American Antiquity 36 (2).
JACKSON, D., 1989. Conservación y microhuellas de uso en instrumentos líticos. Revista Museos 5 y 6.

KEELEY, L., 1980. Experimental determination of stone tool uses; a microwear análisis. The University of Chicago Press.

NUÑEZ, L., 1983. Paleoindio y Arcaico en Chile: Diversidad, secuencia y procesos. Serie Monografías Cuicuilco, Escuela Nacional de Antropología e Historia, México D. F.

ODELL, G., 1980. Butchering with stone tools: Some experimental results. Lithic Technology IX (2).

ODELL, G. y F. ODELL, 1980. Verifying the reliability of lithicuse-wear assessments by 'Blind Test': The low-power approach. Journal of Field Archaeology 7.

THINGHAM, R., G. COOPER, G. ODELL, B. VOYTEK y A. WHITMAN, 1974. Experimentation in the formation of edge damage: A new approach analysis. Journal of Field Archaeology 1.

SEMENOV S. A., 1982. Tecnología prehistórica. Editorial Akal, Madrid.

WILMSEN, E., 1968. Functional analysis of flaked stone artifacts. American Antiquity 33 (2). 